\title{
Experiencias en el Desarrollo de Aplicaciones Móviles con Interfaces basadas en la Interacción Física
}

\section{Experiences in the Development of Mobile Applications with Interfaces based on Physical Interaction}

Pablo Martín Vera. ${ }^{1}$

Rocío Andrea Rodríguez. ${ }^{1}$

Mariano Kaimakamian Carrau .2

${ }^{1}$ Centro de Altos Estudios en Tecnología Informática (CAETI)

2Universidad Abierta Interamericana (UAI)

Av. Montes de Oca 745, Ciudad Autónoma de Buenos Aires, Argentina 


\section{Resumen}

El hardware de los dispositivos permite desarrollar aplicaciones más complejas que aprovechen los sensores de los mismos en beneficio de sus usuarios. Este artículo presenta diversas aplicaciones desarrolladas basadas en el uso de uno o más sensores disponibles, abriendo un panorama amplio a nuevas maneras de interactuar con los dispositivos. Las interfaces físicas permiten interactuar de forma más natural e intuitiva, moviendo, girando o simplemente apoyando el dispositivo sobre una superficie. Se podrá evidenciar como los desarrollos realizados proponen nuevas formas de interacción.

Palabras clave. Interfaces Físicas, Dispositivos Móviles, Sensores, Sensor de Proximidad, NFC, GPS, Brújula, Barómetro.

\section{Abstract}

The hardware of the devices allows the development of more complex mobile applications that take advantage of their sensors for the benefit of their users. This article presents different applications developed that are based on the use of one or more available sensors, opening a broad panorama to new ways of interacting with devices. The physical interfaces allow for interacting in a more natural and intuitive way, moving, rotating or simply resting the device on a surface. It will be able to demonstrate how the developments made propose new forms of interaction.

Keywords: Physical Interfaces, Mobile Devices, Sensors, Proximity Sensor, NFC, GPS, Compass, Barometer.

\section{Introducción}

Los dispositivos móviles han tenido una alta inserción en el mercado, sobre todo el teléfono celular. Llama la atención ver que hay más cantidad de líneas activas que de habitantes. "Según CICOMRA (Cámara de Informática y Comunicaciones de la República Argentina) en la actualidad en Argentina se encuentran unos 57.850.200 teléfonos celulares en servicio (con líneas activas), (CICOMRA, 2014). Esa cantidad de equipos supera ampliamente la cantidad de habitantes del país. Tomando como basamento Octubre del 2010: según el INDEC la cantidad de habitantes era de 40.117.096 (INDEC, 2010) y la cantidad de celulares con líneas activas era de 56.624.200 (CICOMRA, 2014). Por lo que la penetración del servicio de telefonía móvil es aproximadamente $141,15 \%$ (cantidad de líneas activas/cantidad de habitantes x 100)" (Giulianelli, Pons, Vera, et.al., 2012).

Los teléfonos de gama media y principalmente los de alta (smartphones) poseen una gran cantidad de componentes y sensores (ver figura 1). 


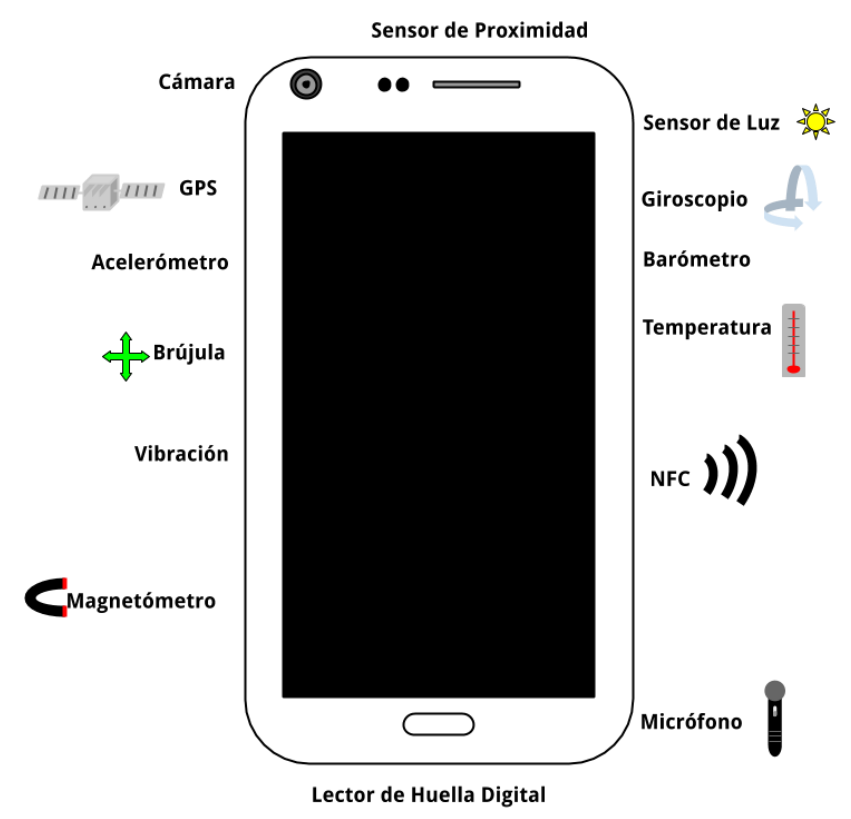

Figura 1. Componentes y Sensores de un Smartphone

El hardware de estos dispositivos permite realizar distintos tipos de aplicaciones las cuales pueden aprovechar los sensores de los dispositivos. Actualmente con HTML 5 (W3Schools, 2019) y las APIs del W3C (Consorcio Web a nivel internacional) (W3C, 2017) es posible realizar desarrollos que accedan al hardware lo cual previamente era únicamente posible desde soluciones nativas (desarrolladas para un sistema operativo en particular) (Rodríguez, Vera, Martinez, et.al., 2014). Estos componentes y sensores podrán ser de gran utilidad al momento de querer realizar nuevas interfaces que sean más naturales e intuitivas.

\subsection{Nuevas Interfaces}

No sólo las pantallas táctiles permiten interactuar de una forma distinta con las aplicaciones, sino que también el conjunto de sensores y componentes que poseen permitirán generar nuevas formas de interacción, las cuales son más naturales y simplifican las acciones del usuario.

La Real Academia Española (RAE) define a la interfaz de dos formas distintas:

- "Conexión o frontera común entre dos aparatos o sistemas independientes" (RAE, 2019).

- "Conexión, física o lógica, entre una computadora y el usuario, un dispositivo periférico o un enlace de comunicaciones" (RAE, 2019).

La segunda definición es la que la RAE propone para el ámbito informático que agrega al usuario como parte de la posible conexión planteada. Si bien las definiciones son generales, es necesario considerar que se ha avanzado poco a poco a un cambio conceptual en las interfaces y deja de ser tan sólo si la interfaz es gráfica (GUI - Interfaz gráfica de Usuario) o de línea de comando, apareciendo múltiples opciones entre ellas: interfaces por: voz (lo cual es muy común sobre todo en teléfonos celulares), gestos (muy usado por ejemplo en celulares y consolas de juegos)... la elección de la interfaz debe ser seleccionada según las características de la aplicación, las del dispositivo sobre el cual correrá e incluso las características de sus usuarios (si es que no está dirigida a un público en particular). Si bien en su mayoría las interfaces han mejorado la forma de interacción, algunas muy vinculadas a un hardware específico, continúan presentando dificultades en su uso. Un claro ejemplo es el manejo de un Smart-tv desde el control remoto, si bien en algunos casos se ha incorporado el manejo por voz, en la mayoría de los controles los usuarios deben desplazarse por medio de botones sin un mouse, ni pantalla táctil haciendo muy difícil por ejemplo escribir un texto para realizar una búsqueda. Del mismo modo en un Smartwatch al ser la pantalla reducida son limitadas las acciones que el usuario puede hacer desde dispositivo y también la información a mostrar ya que poseen un área de visualización 
muy reducida. En estos casos puede recurrirse a tener una aplicación (app) que le permita al usuario desde otro dispositivo (ej: celular, tablet, etc...) visualizar la información de configuración o bien descargar información generada (ej: estadísticas de ritmo cardiaco, cantidad de pasos en el día...). Del mismo modo un celular podría convertirse en un control remoto mediante una app para facilitar el uso de un Smart-tv. En la figura 2 se representa el primer enfoque en donde el usuario tiene un conjunto de acciones a realizar y una primera interfaz que puede ser desde un dispositivo intermedio por ejemplo su teléfono celular y desde allí interactuar con el dispositivo final (por ejemplo Smart-tv, Smartwatch), lo cual no quita que en forma menos amena y hasta en algunos casos de acciones reducidas podría tener una interfaz directa con el dispositivo 2.

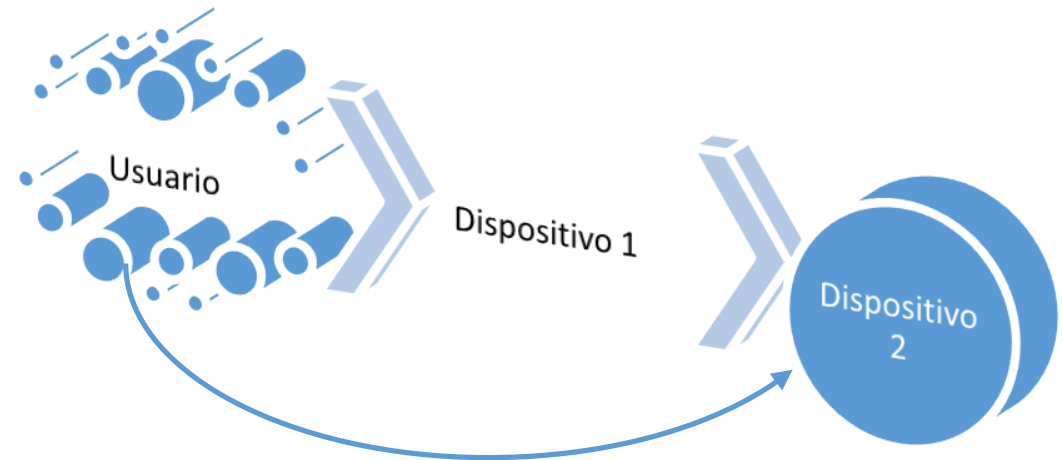

Figura 2. Niveles de interfaces para el acceso al Dispositivo 2, en forma directa o por medio de otro dispositivo

Mucho se menciona sobre las interfaces en cuanto a su diseño, interacción... pero también se cuenta con interfaces que deben estar "ausentes", lo esperado es que el usuario no deba interactuar con la aplicación. A modo de ejemplo, puede plantearse el caso de una alarma conectada en una casa, si la alarma funciona correctamente salvo en el caso de detectar una intrusión la alarma pasará inadvertida. En las redes de área personal BAN (Body Area Network) (Seyedi, Kibret, Lai, et.al., 2013), algo parecido debe suceder, el usuario podría tener un conjunto de dispositivos vestibles (wereable) entre ellos el smartwatch, así como un gran número de dispositivos los cuales pueden estar incorporados en prendas diarias. Si los mismos no sensan ninguna anomalía o condición que deba ser informada, el usuario de dichas prendas debería no notar la presencia de estos sensores. "Actualmente se dispone de prendas inteligentes capaces de analizar la frecuencia cardiaca, las alteraciones en el ritmo y la morfología del ECG (Electrocardiograma)" (Pérez-Villacastín, Gaeta, 2015). "La ropa del futuro llevará incorporada sensores capaces de controlar nuestro estado de salud. De forma inmediata, los datos se transformarán en señales eléctricas que podrán ser enviadas a un ordenador o a cualquier dispositivo móvil, como un teléfono, para que las interprete un médico o el propio usuario" (Guerrero, 2012). En el 2016, una empresa privada en conjunto con la Universidad Ramón Llull (Barcelona, España) diseñaron una remera que según la temperatura corporal, por WIFI se comunica con el aire acondicionado y lo regula (ESeficiencia, 2016). Esto podría en gran escala ser aplicado a batas para pacientes hospitalizados, a adultos mayores que viven solos y tienen dificultad o están distraídos y olvidan regular la calefacción del ambiente, etc. Los puntos negativos serán la disponibilidad de dichas prendas masivamente, así como los costos que puedan tener las mismas. Es importante considerar que en este caso puede no existir una interfaz ni necesidad de conexión directa con el usuario, sino que en cambio pueden comunicarse con otra persona que lo supervise por ejemplo con un médico. En este caso se presenta otro enfoque en el que el usuario tiene el dispositivo vestible del cual puede desconocer como da los datos, que datos aporta o como se interpretan y es otro usuario quién accede a una interfaz en la cual obtiene dichos datos o recibe notificaciones y alertas (ver figura 3). 


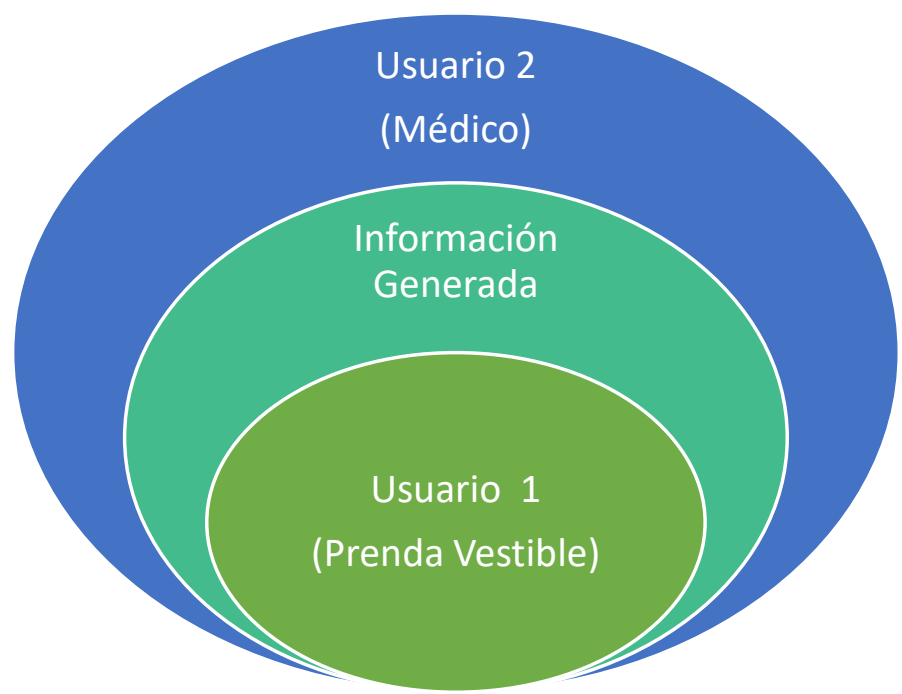

Figura 3. Dos usuarios del sistema uno provee datos sin necesitar conocer el sistema ni interactuar con él y el otro usuario en el nivel final es quién actúa con una aplicación en otro dispositivo (distinto al que genera la información, en este caso sensores incorporados en dispositivos vestibles).

En este enfoque en el que por ejemplo el usuario cuenta con un smartwatch, la tecnología estaría consigo en todo momento y lugar, como omnipresente. Y se la suele denominar "computación ubicua" (Caserotto, 2012), en donde los sensores están empotrados en la ropa del usuario siendo completamente transparente y no modificando los hábitos del mismo.

\subsection{Interfaces con Interacción Física}

Las interfaces con interacción física son aquellas en las que los movimientos de un usuario desencadenan una acción particular, por ejemplo, girar el celular en un sentido u otro para aumentar o disminuir la cantidad de minutos en un temporizador (ver figura 4), Esta aplicación debe poder controlarse de dos formas:

- De forma tradicional utilizando la pantalla táctil arrastrando la aguja al tiempo deseado.

- Sin necesidad de tocar la pantalla (por ejemplo, para cuando la persona está cocinando y tiene las manos sucias). Este segundo modo solo se habilitará cuando el dispositivo esté apoyado sobre una superficie plana y se configurará girándolo. Una vez que se detecte la finalización del movimiento se le dará un tiempo al usuario (por ejemplo 5 segundos) y se comenzará la cuenta atrás automáticamente. 


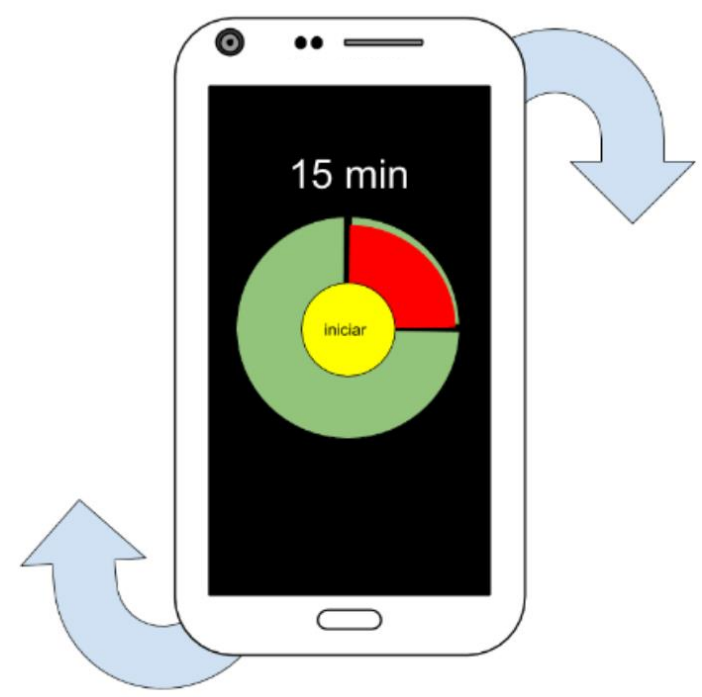

Figura 4. Ejemplo de interfaz para un temporizador con interacción física (controlada mediante sensores de movimiento)

No sólo utilizar los sensores del dispositivo constituye el campo de las interfaces con interacción física, también deben considerarse los gestos. Motorola en algunos de sus teléfonos celulares incorpora gestos para activar la cámara de fotos, la linterna y controlar otras características; de este modo los usuarios eligen si activar o no dichos gestos pudiendo de forma rápida realizar las funcionalidades. En este ejemplo está interfaz con interacción física requiere que el usuario active los gestos y los recuerde, pero es una alternativa ágil para acceder a dichas tareas. Se puede decir que existen dos interfaces aquella que se basa en buscar la aplicación puntual dentro de las pantallas y aquella que se genera como atajo por medio de gestos. Un claro ejemplo de interfaces con interacción física está dado por el sensor Kinect de Microsoft incorporado inicialmente en su consola de videojuegos XBOX y capaz de reconocer los movimientos del cuerpo de una persona. Este sensor ha dado origen a diversos proyectos académicos por ejemplo aplicados a niños con TEA (Transtorno del Especto Autista), en donde se puede trabajar con diversos aspectos cognitivos e incluso de socialización, con pequeñas aplicaciones construidas para este fin (Rodríguez Rodríguez, 2017), (Contreras, 2019).

\section{Contexto}

La Universidad Abierta Interamericana (UAI) realiza actividades de vinculación para acercar a todos los niveles educativos a la tecnología y también a la investigación académica. Esto se genera a través de diversos eventos, como por ejemplo: la Semana de la Ciencia y la Tecnología (al que se unen diversas instituciones a lo largo de Argentina) (UAI, 2018), el CIITI (Congreso Internacional de Innovación Tecnológica) (UAI, 2019) ... también por medio de visitas abiertas que se realizan a los laboratorios del CAETI (Centro de Altos Estudios en Tecnología Informática), ver figura 5. Acercar la tecnología a la comunidad académica desde el nivel primario hasta la universidad, incluyendo también público en general en diversas actividades es un desafío que la Universidad se propone todos los años. Desde el laboratorio de Ingeniería de Software en lo que respecta a los proyectos vinculados con dispositivos móviles, surge una primera aplicación que será presentada más adelante en este artículo que ha sido utilizada en uno de estos encuentros en la que se hace uso del sensor de proximidad acercando el conocimiento a los niños más pequeños mediante un truco de magia, esto ha sido el puntapié inicial para luego generar una aplicación que permita navegar la web a través de gestos realizados en forma aérea detectados por el sensor de proximidad (lo cual se describirá en sección 2.1 del presente artículo). Luego se ha trabajado con la tecnología NFC (Near Field Communication) (NFC.org, 2017), que será abordada en la sección 2.2, para realizar eventos de proximidad lo que ha dado por resultado construir un tablero de llamadas rápidas. 
También se cuenta con un desarrollo, el cual se está mejorando actualmente, de un prototipo para el monitoreo de personas mayores con necesidades especiales por medio de una aplicación que dispara alertas ante situaciones de peligro. Esta aplicación denominada VIGIA permite utilizar diversos sensores del dispositivo de forma no invasiva y preventiva (se presenta en forma resumida en la sección 2.3). Es por eso que este artículo presenta un compendio de las aplicaciones desarrolladas, que permitirá hacer énfasis en el uso de sensores generando interfaces físicas más intuitivas e incluso en algunos casos como lo es en la aplicación VIGIA más inmersivas en donde el usuario puede no detenerse a pensar en la aplicación en sí ya que la misma debe ser transparente sin interrumpir con su vida cotidiana. Es decir, las aplicaciones pueden tener objetivos tan diversos como entretener a niños por medio de la magia, que los lleve a indagar cómo se logra el resultado y descubrir la existencia de sensores, como acompañar a personas en su vida cotidiana sin invadirlos e inferir en sus tareas. Las interfaces deben favorecer la comunicación de los usuarios con la aplicación, por lo cual deben diseñarse las aplicaciones móviles tomando en cuenta los perfiles y necesidades particulares de los usuarios, así como las pantallas reducidas y otras limitaciones que pudieran tener los dispositivos en los cuales serán utilizadas.

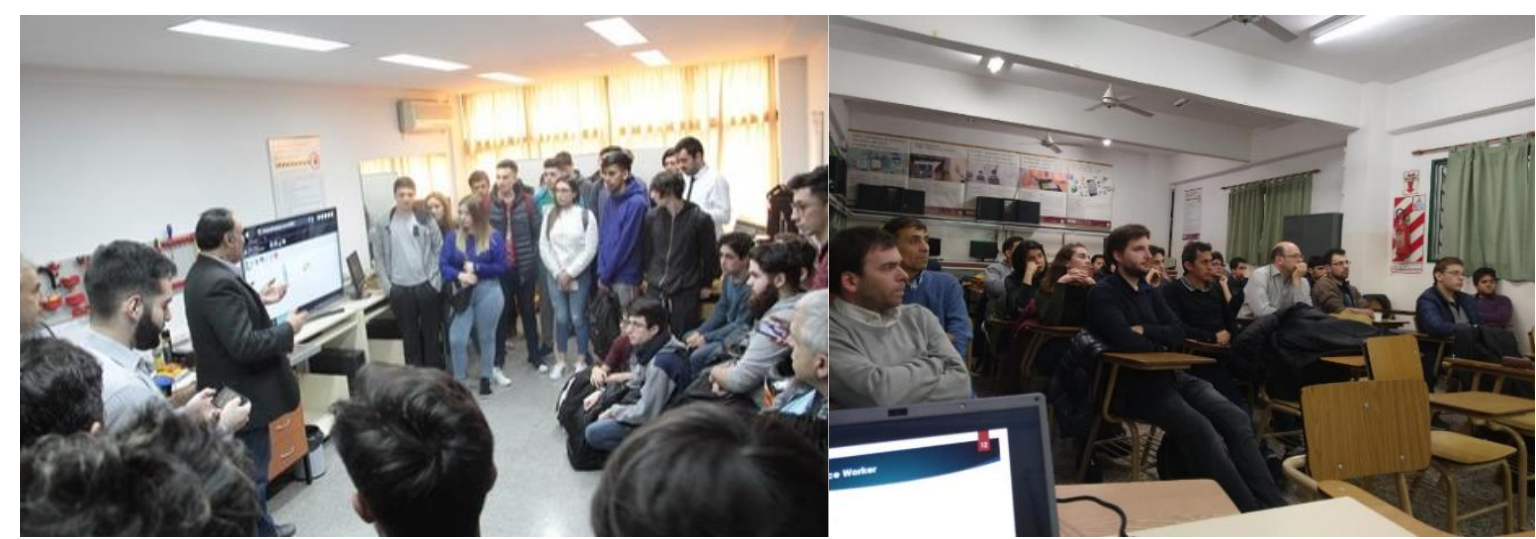

Figura 5. A la izquierda visita a los laboratorios del CAETI, a la derecha curso de capacitación para docentes y alumnos en Sede Oeste (Castelar) - Argentina.

\section{Trabajos Relacionados}

Existen diversos trabajos que aprovechan el hardware de los dispositivos móviles, entre ellos volcados a la enseñanza para la simulación de laboratorios sobre todo utilizado en el campo de física, que permiten tomar mediciones de magnitudes por ejemplo la velocidad angular. Algunos autores presentan incluso la creación de un laboratorio móvil basado en los sensores del celular (Gonzalez, 2014), (Castro, 2013). El uso de sensores y otros componentes de los dispositivos móviles se ha extendido a otras áreas como, por ejemplo, en el campo de la salud para el monitoreo constante de los parámetros de un paciente a través de un dispositivo móvil, como ser un teléfono, entre los trabajos en esa área con desarrollo en Android se encuentran (Kong, 2016), (Robledo, 2015). También se proponen soluciones con el mismo enfoque utilizando dispositivos vestibles como puede ser un reloj (smartwatch), entre dichos trabajos se encuentran los de (Kulkarni,2016), (Jiang,2011). 
Distintos autores abordan la temática de las interfaces físicas, también conocidas como interfaces tangibles (por sus siglas en ingles TUI). Entre los artículos que exploran las consideraciones para este tipo de interfaces se encuentra (Quin, 2009) que plantea métodos interactivos de diseño de productos que intentan romper la barrera establecida por la interfaz gráfica de usuario tradicional, vinculando la acción de copiar y pegar elementos con un instrumento físico. Para manejar una computadora o un determinado equipamiento aparecen este tipo de interfaces, como así también para el campo de la enseñanza "el uso de las Interfaces de usuario tangibles (TUI) como tecnología educativa ha ganado un interés sostenido a lo largo de los años con un acuerdo común sobre su capacidad innata para involucrar e intrigar a los estudiantes en pedagogías de aprendizaje activo" (Attard, 2019). En esta área mucho de lo que se aborda es con una mesa de trabajo física sobre la cual apoyan objetos físicos que son reconocidos y digitalizados (Bonillo, 2019), (Reyes, 2019), (Attard, 2019).

Más allá de las aplicaciones concretas el manejo de la interfaz por medio de gestos es un área de gran importancia, entre otros trabajos (Gupta, 2016), (Murao, 2011), (Trong, 2019) abordan la temática sobre el sistema operativo Android.

Todos los trabajos mencionados tienen puntos de contacto con el presente artículo. A continuación, se presentarán las soluciones desarrolladas por nuestro grupo de investigación: 2 vinculadas con el sensor de proximidad, 1 basada principalmente con el uso de NFC y 1 que integra el uso de diversos sensores.

\section{Soluciones desarrolladas}

Cada una de las soluciones desarrolladas a partido de la base de incorporar uno o más sensores para el uso de las mismas, es por ello que cuentan con interfaces con interacción física. Algunas de las interfaces tan sólo requieren realizar un gesto aéreo identificado detectado por el sensor de proximidad o apoyar el celular sobre una base con etiquetas NFC, otras en cambio, consideran en su desarrollo un conjunto de sensores disponibles en los smartphones. En esta sección se presentan los sensores utilizados para las distintas aplicaciones creadas.

\subsection{Sensor de Proximidad}

Se describe a continuación dos aplicaciones desarrolladas haciendo uso del sensor de proximidad:

1. Mago Colorido: Se desarrolló una aplicación web a la que se denominó Mago Colorido, en la que según el tiempo de detección de obstrucción del sensor de proximidad muestre un color en la pantalla. ¡Elijan un color! A partir de allí los niños pequeños pudieron evidenciar esta especie de truco de magia. Los únicos colores seteados eran: Rojo, Verde, Azul, Amarillo y Rosa. La persona que tiene el teléfono celular (el mago) acerca su mano a la pantalla del teléfono y hace aparecer el color en la pantalla. Para esto la aplicación da un feedback mediante vibración, de forma tal que una vibración identifica un color, dos vibraciones otro y así sucesivamente, tan solo debe acordarse el orden de los colores. No se setearon más colores dado que el tiempo total para esperar al último color aumentaría significativa, así como la posible falla del mago por no recordar el orden de los colores, fue por eso que se hizo una encuesta entre los conocidos del grupo de investigación para probar los colores que se repiten con más frecuencia. Llamativamente no sólo los niños sino las personas adultas dicen inicialmente uno de los 4 colores primeramente seteados apenas se les consulta, a lo cual luego se agregó el rosa. Ver el asombro en los rostros de los niños e intentar decir otro color para ver si también funcionaba es una forma de medir el éxito de esta pequeña aplicación. Se ha probado también con personas más grandes que ya al segundo intento ofrecen otras ocurrencias de colores: Bordo, fucsia, Naranja, etc. Para esto se configuró una página que decía no tengo ese color. La motivación es que el público se pregunte ¿cómo lo logran?, de hecho, con público adulto en general no asociado a la tecnología, nos ha ocurrido que presuponen que el truco se efectúa por medio de la voz, hasta nos han escrito el color en un papel para que el dispositivo "no los escuche". Esta 
pequeña aplicación que permite descubrir un sensor del dispositivo que puede explicarse claramente a los niños y al público en general, ya que es el mismo que se utiliza convencionalmente cuando se apaga la pantalla al momento de llevar el teléfono celular a la oreja para atender un llamado, despierta la curiosidad en los muchos usos que se le puede dar a los sensores del dispositivo. Por otra parte, el desarrollo realizado para lograr esto permitió investigar en mayor medida sobre el funcionamiento del sensor y trasladar el conocimiento a otro tipo de aplicaciones.

2. Gestos de Proximidad: Con la base de experiencia previa en el uso del sensor de proximidad, se analiza más a fondo la tecnología para construir una nueva solución. Lo primero a saber es que el sensor de proximidad sólo mide si hay presencia de un objeto cercano ó no (por ejemplo: la oreja al realizarse una llamada), pero no mide distancia de dicho objeto con respecto al sensor. Los valores arrojados por el sensor es 0 cuando detecta un objeto ó superior cuando no lo detecta (ver figura 6).

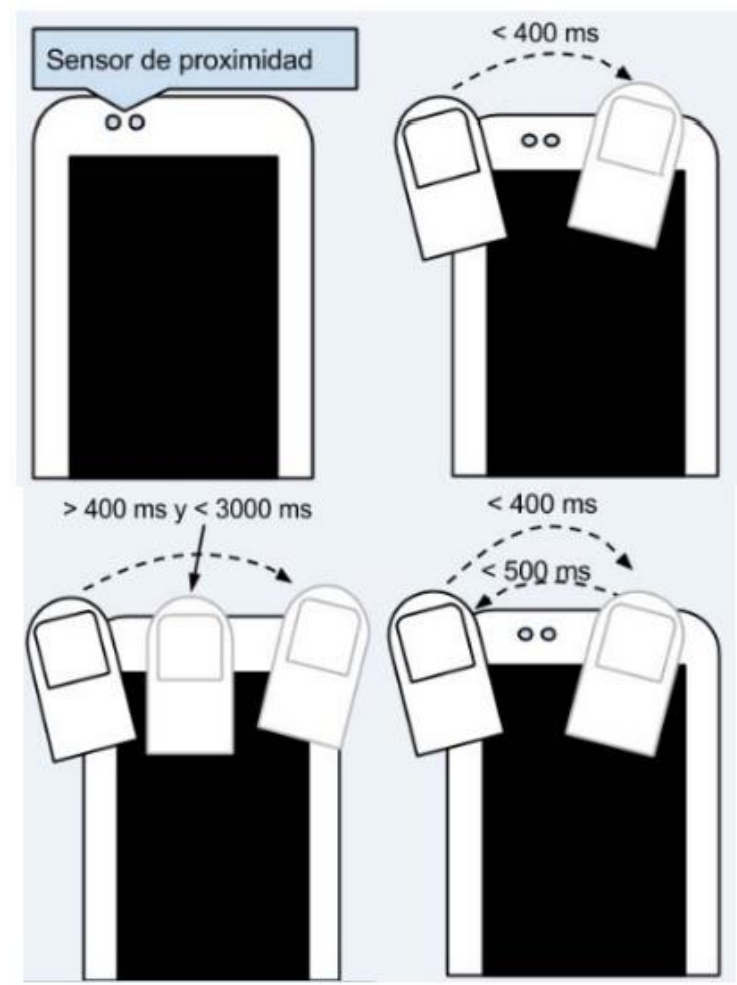

Figura 6. Tiempos configurados para la detección de pases sobre el sensor de proximidad

Se desarrolló una función en JavaScript que permite capturar eventos según si se obstruye el sensor o se libera, lo que permite pasar la mano o un dedo sobre el sensor y que este lo note y pueda reaccionar al respecto. Se creó una API que permite detectar 3 tipos de gestos: pase corto, pase largo ó pase doble (ida y vuelta), se realizaron diversas mediciones de tiempos que dependieron de dos factores: (1) reacción y velocidad de los usuarios (en lo que diferencia a un pase corto de uno largo), (2) exactitud del sensor, dado que entre los diferentes equipos hay sensores con detección más precisa que otros. Esto origino como resultado una publicación académica en el IEEE (Vera, Rodríguez, 2016) con los resultados de la prueba y la configuración de los tiempos que permitieron que la aplicación funcione correctamente más allá del usuario y de la calidad del sensor de proximidad del equipo utilizado.

Teniendo disponible este sensor y la función para detectar los eventos se proponen entonces implementar las siguientes ayudas a la navegación:

- Utilizar la doble pasada: por ejemplo, para mostrar en un menú desplegable las opciones de navegación, esto evitará tener que hacer scroll para moverse hasta donde se encuentre el menú o el ícono que abre el mismo. El menú desplegable podrá a su vez cerrarse mediante una pasada corta. Otra opción sino se desea utilizar en menú 
desplegable es que la doble pasada lleve automáticamente a la parte superior de la pantalla donde se encuentran las opciones.

- Utilizar la pasada larga: para volver al "home" del sitio web. Creando un acceso directo desde cualquier lugar del sitio para ir a la página principal. Estos ejemplos de uso dejan en evidencia las alternativas adicionales que se le ofrecen a los usuarios finales, mediante el uso del sensor de proximidad.

Como puede observarse estas facilidades adicionales a la interfaz de la aplicación constituyen lo que se denomina una interfaz física, el usuario realiza gestos en el aire (cerca del sensor de proximidad) lo cual denominamos en forma general gestos de proximidad y mediante dichos gestos desencadena acciones de forma rápida que le evitarán por ejemplo hacer scroll en una página para regresar a la parte superior.

\subsection{NFC}

NFC (Near Field Communication) es una tecnología inalámbrica que permite transferir datos entre dispositivos que estén próximos entre sí (aproximadamente a 10 centímetros de distancia). La frecuencia de transmisión es de $13,56 \mathrm{MHz}$ (no requiere de licencia para su uso) (NFC FORUM, 2013). La comunicación se realiza entonces cuando se cuenta con dos dispositivos con NFC (Rodríguez, Vera, Martínez, et. al., 2013):

- Activo: El dispositivo genera un campo electromagnético y este se usa para establecer la información leyendo datos o trasmitiendo datos al otro dispositivo

- Pasivo: Sin posibilitar el campo electromagnético, tienen información que un dispositivo activo podrá leer o modificar.

Existen distintas soluciones implementadas que utilizan NFC, en Argentina la tarjeta SUBE en la cual se carga crédito que permite pagar los pasajes del transporte público posee un tag NFC. Es una tecnología simple de implementar y los tag de NFC tienen bajo costo. La tarjeta actúa como dispositivo pasivo. Son dispositivos activos las máquinas que tiene cada trasporte público que leerán los datos de la tarjeta restarán el importe del viaje al crédito existente, etc... así también lo son los teléfonos con NFC, muchos usuarios tienen descargada la aplicación que permite ver el saldo de sus tarjetas, cargarles crédito, etc...

Se desarrolló una solución particular utilizando esta tecnología:

1. Tablero NFC: planificado para poder realizar llamadas rápidas, como si fuera una agenda de contactos en donde con sólo apoyar el teléfono en la fotografía de una persona se dispara el llamado. Esto podría ser utilizado por cualquier persona que quiera realizar una llamada en forma rápida, es sumamente útil para adultos mayores no familiarizados con la tecnología, así como para personas no videntes que requieren de un programa lector que las asista para realizar un llamado con el teléfono celular, podrían realizar una llamada rápida apoyando el celular en un tablero señalizado en braile. Pero no implica que la solución sea realizada exclusivamente para estos casos, imaginemos un empleado corporativo que tiene el teléfono celular, otorgado por la empresa para cuestiones laborales y que tiene junto a su estación de trabajo un tablero NFC con los contactos a los que debe llamar frecuentemente. Ofrecer una alternativa rápida de interactuar con un dispositivo es algo que pueden aprovechar todos los usuarios. 


\subsection{Utilización de diversos sensores}

Se desarrolla una aplicación que integra distintos sensores que posibilitan monitorear a un usuario, inicialmente fue desarrollada para teléfonos celulares con Android (los prototipos iniciales con sus pantallas traducidas a inglés fueron presentados en un congreso del IEEE (Vera, Kaimakamian Carrau, Rodríguez, 2018) y luego migrada a Wear OS para ser utilizada desde un smartwatch. La conveniencia de su migración es que resulta más transparente, al usuario además las tecnologías vestibles como lo son el smartwatch aseguran que siempre tendrán consigo el dispositivo en este caso el reloj, por el contario un teléfono celular podría ser apoyado sobre una mesa mientras la persona se desplaza. "La utilización de tecnologías del tipo "vestible" ha aumentado notablemente en los últimos años, permitiendo medir distintas variables fisiológicas, ambientales y de movimiento, a través de dispositivos anexados a la muñeca y/o la ropa del usuario" (Ponce, Lopez, Piccini, et. al., 2016).

La aplicación fue programada en Android, se utilizó SQL Lite y se desarrolló mediante una arquitectura de capas, que permiten separar la funcionalidad de la interfaz de usuario para luego poder adaptarla y migrarla a un dispositivo vestible basado en Android.

Esta aplicación utiliza diversos sensores entre ellos: GPS, Brújula, Acelerómetro, Barómetro, NFC, a continuación, se describe cómo surge y brevemente el funcionamiento de la misma.

1. Vigia: Cuando se crea esta aplicación se pone el foco en personas que requieran un monitoreo particular y no vivan acompañadas, de forma tal que no pueden ser monitoreadas. Es ideal para personas que requieran asistencia (Ej: Personas con discapacidad, Adultos mayores, etc.), es importante comprender que cada persona tiene necesidades particulares que deben ser consideradas. Las dificultades que puedan presentarse pueden estar dentro de sus viviendas, así como cuando salen a hacer compras o realizan un paseo, pudiendo perder el sentido de la orientación. Las tecnologías móviles pueden proporcionar soluciones para estos problemas, generando un sistema de control no invasivo que puede ser monitoreado por familiares y amigos. Sucede en muchos casos que las personas se encuentran restringidas a no salir de un radio sin un acompañante por temor a que algo pueda ocurrirles, este sistema les daría cierta libertad pudiendo informar en tiempo real de lo que se detecte que pueda causar dificultad en la persona monitoreada. Vigia es una aplicación integral en la que es posible configurar que tipo de alarmas se generarán en base a las necesidades de monitoreo particulares, así también el o los destinatarios de los avisos emitidos por esas alarmas. La aplicación utiliza los sensores en dispositivos como:

- GPS, brújula, acelerómetros: Estos sensores en conjunto permiten saber por ejemplo si la persona se aleja de un radio predefinido, en qué dirección lo hace, con qué velocidad lo que permite conocer si está viajando en algún transporte.

- Barómetro: Permite conocer la altitud a la que se encuentra, por ejemplo, una persona que tiene problemas en las rodillas y no debe subir ni bajar escaleras podría alertar si la persona está en un sótano o en la terraza de la vivienda.

- NFC: Que permite tener un registro de la medicación que la persona va tomando. Si se utiliza un simple sistema de alarmas para avisar que se debe tomar un medicamento, la persona puede posponer la alarma o apagarla y omitir luego tomar la medicación. La incorporación del NFC genera el hábito de que cada vez que toma una medicación cargar a través de un tag NFC al sistema que ya tomó la medicación. Este tag se podría pegar en la caja del medicamente para obligar a la persona a moverse donde está el medicamento para apagar la alarma reduciendo la posibilidad de que no la ingiera.

El buen uso de esta aplicación puede ayudar a mejorar la calidad de vida y así también poder guardar registro de lo ocurrido para poder establecer estadísticas y parámetros que puedan contribuir a que el personal médico conozca mejor al paciente. 


\section{Limitaciones}

Todas las aplicaciones mencionadas hacen uso del hardware presente en una gran variedad de dispositivos móviles actuales, sin embargo, en el mercado aún existen equipos que no disponen de los sensores necesarios. Un claro ejemplo es la disponibilidad de NFC que en ciertos mercados como el europeo se utiliza en gran escala, pero en mercados latinoamericanos, al no utilizarse tan frecuentemente, los fabricantes no los incluyen en las versiones regionales para abaratar costos. A modo de ejemplo en la figura 7 se muestra una captura de pantalla tomada de la página gsmarena.com. Esta figura muestra una parte de las especificaciones del celular Motorola Moto G8 plus donde puede verse que el sensor NFC está disponible dependiendo del mercado al cual esté enfocado.

\begin{tabular}{lll}
\hline COMMS & WLAN & Wi-Fi $802.11 \mathrm{a} / \mathrm{b} / \mathrm{g} / \mathrm{n} / \mathrm{ac}$, dual-band, Wi-Fi Direct, hotspot \\
& Bluetooth & 5.0, A2DP, LE, aptX \\
GPS & Yes, with A-GPS, GLONASS, BDS, GALILEO \\
NFC & Yes (market dependent) \\
Radio & FM radio \\
USB & 2.0, Type-C 1.0 reversible connector, USB On-The-Go
\end{tabular}

Figura 7. Características técnicas del Motorola Moto G8 plus

Otro de los problemas y limitaciones que se pueden encontrar es el versionado del sistema operativo. Frecuentemente equipos que tiene el hardware suficiente y los sensores por cuestiones comerciales no reciben actualizaciones en la versión del sistema operativo. Esto puede ser un inconveniente a la hora de desarrollar ya que ciertas APIs y funcionalidades que facilitan el uso de los sensores se van incorporando en los SDKs de las nuevas versiones de los sistemas operativos móviles haciendo que no puedan utilizarse en versiones anteriores 0 requieran de un desarrollo mucho más laborioso y tedioso.

Como último punto podemos nombrar que para las aplicaciones de monitoreo lo ideal es utilizar tecnologías vestibles como por ejemplo un smartwatch. Estos equipos aún no están lo suficientemente masificados y son costosos. Además, existen diversas marcas y algunas con sistemas operativos propietarios y de poca difusión. Es por eso que se eligió Android para el desarrollo ya que se puede utilizar en celulares y luego portar la solución a equipos con WearOs adaptando la interfaz.

\section{Conclusiones}

Existe una gran variedad de aplicaciones que pueden verse favorecidas con el uso del hardware actualmente disponible en los dispositivos móviles, conocer el funcionamiento de los distintos sensores permitirá desarrollar aplicaciones más especializadas. En este artículo se presentaron diversas aplicaciones con objetivos muy distintos que plantean diferentes formas de interacción desde los gestos aéreos al pasar la mano cerca del sensor de proximidad, hasta apoyar simplemente el dispositivo sobre una superficie para lanzar un evento como lo es una llamada por medio del tablero NFC o bien una aplicación más inmersiva la cual no requiere comunicación alguna con el usuario al que directamente protege, sino que lanza alertas a personas designadas en el círculo íntimo del usuario en caso de detectar riesgos. Este último desarrollo inició como un prototipo desde el teléfono celular, para ser migrado luego a un smartwach para que sea más inmersivo. La tecnología puesta al servicio de las personas ha sido el eje de este artículo enfocado en nuevas interfaces las cuales mediante interacción física permiten establecer otros esquemas de interacción. 


\section{REFERENCIAS}

Attard, G., De Raffaele, C., \& Smith, S. (2019). TangiBoard: a toolkit to reduce the implementation burden of tangible user interfaces in education.

Bonillo, C., Marco, J., Baldassarri, S., \& Cerezo, E. (2019). KitVision toolkit: supporting the creation of cognitive activities for tangible tabletop devices. Universal Access in the Information Society, 1-29.

Caserotto, G. A. (2012). Computación ubicua, sensibilidad al contexto y mashups (Tesis Especialita en Ingeniería de Software, Facultad de Informática, Universidad Nacional de La Plata).

CICOMRA - Cámara de Informática y Comunicaciones de la República Argentina (2011). Estadísticas - Telefonía Móvil, Argentina

http://www.cicomra.org.ar

Contreras, V. H. (2019). Interfaces Gestuales: Herramienta innovadora para complementar el aprendizaje en niños con TEA (Tesis Especialista en Ingeniería de Software, Universidad Nacional de La Plata).

ESeficiencia: Portal de Eficiencia y Servicios Energéticos. (2016). Desarrollan ropa inteligente conectada a la climatización del hogar.

https://www.eseficiencia.es/2016/09/16/desarrollan-ropa-inteligente-conectada-a-la-

climatizacion-del-hogar

Giulianelli, D. A., Pons, C., Vera, P. M., Rodríguez, R. A., Trigueros, A., Fernández, V., \& Marko, I. (2012). Técnicas para la validación de un sitio web móvil. In XIV Workshop de Investigadores en Ciencias de la Computación.

Guerrero Teresa, (2012). Ropa 'inteligente' para vigilar el estado de salud. https://www.elmundo.es/elmundo/2012/05/03/nanotecnologia/1336065302.html

González, M. Á., González, M. Á., Llamas, C., Martín, M. E., Vegas, J., Martínez, Ó., ... \& Herguedas, M. (2014, October). Mobile phones for teaching physics: using applications and sensors. In Proceedings of the Second International Conference on Technological Ecosystems for Enhancing Multiculturality (pp. 349-355).

Gupta, H. P., Chudgar, H. S., Mukherjee, S., Dutta, T., \& Sharma, K. (2016). A continuous hand gestures recognition technique for human-machine interaction using accelerometer and gyroscope sensors. IEEE Sensors Journal, 16(16), 6425-6432.

INDEC (Instituto Nacional de Estadísticas y Censos), Datos Definitivos-Censo 2010, Argentina, (2010)

https://www.indec.gob.ar/indec/web/Nivel3-Tema-2-41

Kong, X., Fan, B., Nie, W., \& Ding, Y. (2016, October). Design on mobile health service system based on Android platform. In Advanced Information Management, Communicates, Electronic and Automation Control Conference (IMCEC), 2016 IEEE (pp. 1683-1687). IEEE.

Kulkarni, C., Karhade, H., Gupta, S., Bhende, P., \& Bhandare, S. (2016, January). Health companion device using loT and wearable computing. In Internet of Things and Applications (IOTA), International Conference on (pp. 152-156). IEEE.

Murao, K., Terada, T., Yano, A., \& Matsukura, R. (2011, June). Evaluating gesture recognition by multiple-sensor-containing mobile devices. In 2011 15th Annual International Symposium on Wearable Computers (pp. 55-58). IEEE.

NFC Forum (2013). About the technology

https://nfc-forum.org/what-is-nfc/about-the-technology/ 
NFC.org,(2017). Near field communication

http://nearfieldcommunication.org/

Pérez-Villacastín, J., \& Gaeta, E. (2015). ¿Prendas inteligentes para cuidar a las personas o personas inteligentes que utilizan prendas para cuidarse? Revista Española de Cardiología, 68(7), 559-561.

Ponce, S., Lopez, N., Piccinini, D., Roberti, M., Avetta, S., Andino, N., ... \& Garcia, C. (2016). Desarrollo de un sistema vestible de medición de variables fisiológicas. In Proc VII Congreso de Ingeniería Biomédica.

Qin, J., Guan, Y., \& Ji, H. (2009, November). TUI interactive product design. In 2009 IEEE 10th International Conference on Computer-Aided Industrial Design \& Conceptual Design (pp. 14551458). IEEE.

RAE - Real Academia Española (2019). Interfaz.

https://dle.rae.es/?id=Lu1HQaO

Rebolledo-Nandi, Z., Chavez-Olivera, A., Cuevas-Valencia, R. E., Alarcon-Paredes, A., \& Alonso, G. A. (2015, March). Design of a versatile low cost mobile health care monitoring system using an android application. In Health Care Exchanges (PAHCE), 2015 Pan American (pp. 1-4). IEEE.

Reyes-Flores, A., Mezura-Godoy, C., \& Benítez-Guerrero, E. (2019, October). Requirements Analysis for Tangible User Interfaces for Collaborative Use. In 2019 IEEE International Conference on Engineering Veracruz (ICEV) (Vol. 1, pp. 1-7). IEEE.

Rodríguez Rodríguez, A. (2017). Recursos digitales en Educación Especial. Propuesta educativa para centrar la atención de niños con TEA a través del sensor Kinect.

Rodríguez, R. A., Vera, P. M., Martínez, M. R., Pons, C., Valles, F. E., \& de La Cruz, L. V. (2014). Reducing the Gap between Native and Web Applications.

Rodríguez, R. A., Vera, P. M., Martínez, M. R., Giulianelli, D. A., \& Valles, F. E. (2013). Context Aware Applications on Mobile Environments-Engaged by the use of NFC. In International Conference on Multimedia, Scientific Information and Visualization for Information Systems and Metrics (pp. 29-31).

Seyedi, M., Kibret, B., Lai, D. T., \& Faulkner, M. (2013). A survey on intrabody communications for body area network applications. IEEE Transactions on Biomedical Engineering, 60(8), 20672079.

Trong, K. N., Bui, H., \& Pham, C. (2019, October). Recognizing hand gestures for controlling home appliances with mobile sensors. In 2019 11th International Conference on Knowledge and Systems Engineering (KSE) (pp. 1-7). IEEE.

UAI (2018), Semana Nacional de la Ciencia y la Tecnología.

https://noticias.uai.edu.ar/facultades/tecnolog\%C3\%ADa-inform\%C3\%A1tica/xvi-semananacional-de-la-ciencia-y-la-tecnolog\%C3\%ADa/

UAI (2019), Congreso Internacional en Innovación Tecnológica Informática. https://www.uai.edu.ar/ciiti//

Vera, P. Kaimakamian Carrau, M., Rodríguez R. (2018). Mobile follow-up system for elderly and disabled people, Congreso Argentino de Ciencias de la Informática y Desarrollos de Investigación (CACIDI), Buenos Aires, 2018, pp. 1-5.

doi: 10.1109/CACIDI.2018.8584361

Vera, P. M., \& Rodriguez, R. A. (2016). Creating and Using Proximity Events on Mobile Websites. IEEE Latin America Transactions, 14(11), 4579-4584. 
W3C (2017), W3C Mission

https://www.w3.org/Consortium/mission

W3schools (2019), HTML 5 Introduction.

https://www.w3schools.com/html/html5 intro.asp 


\section{NOTAS BIOGRÁFICAS}

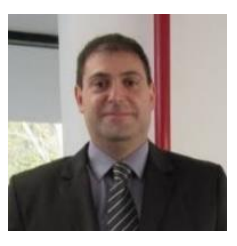

Pablo Martín Vera. Doctor en Ciencias Informáticas (UNLP - Universidad Nacional de La Plata). Ingeniero en Informática (UNLaM - Universidad Nacional de La Matanza). Docente de grado y posgrado en UNLaM, UTN (Universidad Tecnológica Nacional) y UAI (Universidad Abierta Interamericana). Es además director de proyectos de investigación en el CAETI (Centro de Altos Estudios en Tecnología Informática) de la UAI, destacándose entre sus principales áreas de interés la gamificación, las tecnologías móviles, la usabilidad y el desarrollo dirigido por modelos (MDD). En cuanto a la formación de recursos humanos es director de becarios y director de tesis de maestría y doctorado. Es revisor de distintas publicaciones académicas y revistas indexadas.

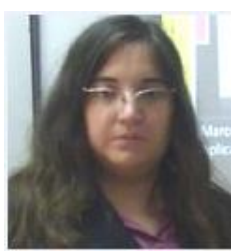

Rocío Andrea Rodríguez. Doctora en Ciencias de la Computación (UNLP-Universidad Nacional de La Plata), Ingeniera en Informática (UNLaM-Universidad Nacional de La Matanza). Profesora de Grado en UNLaM y UTN (Universidad Tecnológica Nacional); profesora de posgrado en UAI (Universidad Abierta Interamericana) y UNLaM. Directora de proyectos de investigación en el CAETI (Centro de Altos Estudios en Tecnología Informática) de la UAI UAI. Además es directora de becarios, pasantes y tesistas. Ha participado como jurado de tesis y es revisora de: artículos, proyectos de extensión universitaria y programas cofinanciados. Es autora de libros, capítulos de libros y artículos académicos.

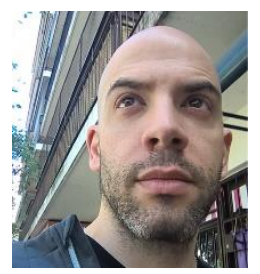

Mariano Kaimakamian Carrau. Técnico Diseñador Multimedial (Escuela Da Vinci), Ingeniero en sistemas informáticos (Universidad Abierta Interamericana). Docente de grado en la Universidad Tecnológica Nacional. Investigador en el CAETI (Centro de Altos Estudios en Tecnología Informática) de la UAI. A nivel laboral, en los últimos diez años se ha desenvuelto en distintas áreas que incluyen el análisis y desarrollo de soluciones informáticas, hasta el liderazgo de equipo y gestión de proyecto; actualmente es arquitecto de software y DevOp, responsable de migraciones de sistemas stand alone a entornos web. Entre sus principales logros se encuentra haber participado en el desarrollo de la plataforma SECF dentro del marco del proyecto espacial nacional de la República Argentina.

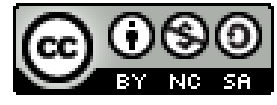

Esta obra está bajo una licencia de Creative Commons Reconocimiento-NoComercial-CompartirIgual 2.5 México. 